\title{
Uno scrittore-viaggiatore: Nicolas Bouvier (1929-1998) e la sua prima biografia
}

\section{Francesca Morale}

\section{(2) OpenEdition}

1 Journals

\section{Edizione digitale}

URL: http://journals.openedition.org/studifrancesi/6168

DOI: 10.4000/studifrancesi.6168

ISSN: 2421-5856

\section{Editore}

Rosenberg \& Sellier

\section{Edizione cartacea}

Data di pubblicazione: 1 novembre 2010

Paginazione: 508-514

ISSN: 0039-2944

\section{Notizia bibliografica digitale}

Francesca Morale, «Uno scrittore-viaggiatore: Nicolas Bouvier (1929-1998) e la sua prima biografia», Studi Francesi [Online], 162 (LIV | III) | 2010, online dal 30 novembre 2015, consultato il 07 janvier 2021. URL: http://journals.openedition.org/studifrancesi/6168; DOI: https://doi.org/ERREUR PDO dans / localdata/www-bin/Core/Core/Db/Db.class.php L.34 : SQLSTATE[HY000] [2006] MySQL server has gone away

\section{(c)}

Studi Francesi è distribuita con Licenza Creative Commons Attribuzione - Non commerciale - Non opere derivate 4.0 Internazionale. 


\section{Uno scrittore-viaggiatore: Nicolas Bouvier (1929-1998) e la sua prima biografia}

Chi era Nicolas Bouvier? Un ginevrino fuori dal comune, uno scrittore dalla raffinatissima espressione, un infaticabile viaggiatore, un fotografo curioso, un documentarista serio ed affidabile. Era nato a Ginevra il 6 marzo 1929, in seno ad una famiglia borghese e fin dall'adolescenza aveva avuto un accesso privilegiato a tutte le espressioni visive, musicali e culturali della Ginevra colta del tempo ${ }^{1}$. Le amicizie di famiglia lo avevano inoltre avvicinato alla cultura delle università tedesche e di quella musicale bavarese; l'occupazione del padre Auguste Bouvier, prima bibliotecario e poi direttore della Biblioteca Pubblica e Universitaria di Ginevra, oggi Biblioteca di Ginevra, lo aveva portato più facilmente a contatto coi libri. Il genitore, che nel tempo libero raccontava ai figli ogni sorta di storie reali e immaginarie - "c'était un conteur tout à fait extraordinaire" ${ }^{2}$ ci dice lo scrittore - aveva contribuito indirettamente alla voglia di viaggiare del Nostro, incoraggiandolo a partire ${ }^{3}$. La madre e la governante prussiana gli avevano impartito invece una rigida educazione calvinista che lo aveva segnato profondamente e che è palpabile in molti suoi scritti, anche se essa è discretamente tenuta sotto controllo da un sottile e garbato umorismo che rese Bouvier molto simpatico agli amici e stimato dal pubblico. Terminati gli studi umanistici al Collège Calvin della sua città e poi quelli universitari con due lauree parallele, una in diritto, l'altra in lettere, il giovane e colto ginevrino, con un'inquietudine sofferta, espressa appieno dai versi di Shakespeare in epigrafe all'Usage du Monde, "I shall be gone and live or stay and dy" ${ }^{4}$ lascia la sua città natale per percorrere quelle strade del mondo che lo tramuteranno in un infaticabile scrittore-viaggiatore. Bouvier attraverso gli innumerevoli viaggi che si alternano a periodi di sedentarietà favorevoli alla produzione letteraria, uscirà da sé per farsi altro, per farsi scrittura. Le parole che suggellano il "récit" del suo primo mitico viaggio in oriente:

comme une eau, le monde vous traverse et pour un temps vous prête ses couleurs. Puis se retire, et vous replace devant ce vide qu'on porte en soi, devant cette espèce d'insuffisance centrale de l'âme qu'il faut bien apprendre à côtoyer, à combattre, et qui paradoxalement, est peut-être notre moteur le plus sûr ${ }^{5}$,

(1) A Ginevra, nella casa della Cour Saint-Pierre, situata nella "vieille ville", Auguste Bouvier soleva invitare gli studiosi e intellettuali stranieri di passaggio. Il giovane Nicolas ebbe così modo di incontrare Marguerite Yourcenar nel periodo in cui la scrittrice preparava, non ancora famosa, i suoi Mémoires d'Adrien, e poi ancora Ian Fleming, Musil, Hermann Hesse e Thomas Mann. Di quest'ultimo Bouvier ci dice che sua madre era «très liée avec la famille de Thomas Mann, elle a beaucoup joué avec ses enfants». N. Bouvier, Euvres, Paris, Gallimard «Quarto», 2004, p. 1255.

(2) N. BouviER, Routes et Déroutes in Euvres, op. cit., p. 1257.

(3) Ibid., p. 1258

(4) N. Bouvier, L'Usage du Monde, préface d'Alain Dufour, Genève, Droz, 1999, p. 7.

(5) Ibid., p. 374. 
estendendo «l'insuffisance centrale de l'âme» a tutto il genere umano, tramutano la sua esperienza di viaggio in una lezione e uno stile di vita che ci ricordano Montaigne e che il Nostro negli anni post-bellici reclamerà con inedita forza e convincimento.

Questo lungo percorso fatto di partenze e movimento e di ritorni e sedentarietà si arresterà solo quando la malattia incurabile che lo ghermisce nell'estate 1997 lo fermerà per sempre nel febbraio dell'anno successivo.

Da allora lo scrittore ginevrino ha continuato a riscuotere interesse nei lettori e apprezzamenti della critica, la città del Lemano gli ha dedicato e continua a dedicargli spazi verbali, visivi e musicali'. E la presentazione della prima biografia di Bouvier in occasione del decimo anniversario della sua scomparsa, ad opera dello scrittore francese François Laut nel gennaio del $2008^{7}$ ne è una riprova. Questa è stata preparata proprio nella città natale dello scrittore, fondata sui documenti d'archivio della Biblioteca di Ginevra, presso la quale la famiglia, donandoli, ha trasferito tutti i suoi manoscritti ${ }^{8}$. Laut, autore di numerosi romanzi tra cui $A \ddot{i}$ (l'amour) del 1994 e TobuBabut del 2006, ci parla nell'Avant-propos delle circostanze personali che lo hanno indotto a scrivere di Bouvier: gli stessi interessi come l'amore per la letteratura, per l'oriente, il Giappone in particolare, dove i due si erano incontrati nel 1997; l'origine ginevrina del ramo materno di Laut, cui si può aggiungere la scoperta di una conoscenza comune, del professore Robert Junod, prozio di Laut, che aveva insegnato al liceo «Calvin» di Ginevra. Queste coincidenze ci inducono a constatare che una forte relazione di identità "esplicita" unisce il biografo al biografato a conferma di quanto affermato dal Lejeune? . E Laut nella prefazione non dice di volerci presentare un Bouvier "intime", "intus et in cute" ${ }^{10}$ ?

La dichiarazione dà a questa biografia la dimensione di "texte référentiel" in quanto essa deve somigliare al vero e il primo biografo di Bouvier vi arriva affidandosi alla lettura delle sue carte.

Siamo dunque di fronte ad uno dei casi contemplati da Philippe Lejeune: «temoignage avec prétention à la biographie» nel quale il narratore che ha conosciuto il suo autore, vuole darne conto ai futuri biografi e si affida al materiale manoscritto e bibliografico cui ha avuto accesso restituendoci «une première version de cette biographie» ${ }^{11}$.

Con il titolo, molto significativo, L'œil qui ecritt ${ }^{12}$, Laut coglie il senso della vita e dell'opera di Bouvier, offrendoci nella sua totalità l'essenza del lavoro letterario dello scrittore, perseguito con costante dedizione fino alla fine della sua non lunga vita ${ }^{13}$. Un corposo materiale fotografico, oggi custodito al Musée de l'Elysée di Losanna,

(6) Citiamo Le Vent des routes. Entretiens avec et autour de Nicolas Bouvier, Genève, Editions Zoé et Radio Suisse- Romande 2005. Pubblicazione che è corredata da due $C D$ riproducenti le registrazioni musicali che Bouvier aveva effettuato durante il suo viaggio in oriente con Thierry Vernet. Nel gennaio 2009 un'esposizione presso la Pinacothèque des Eaux Vives di Ginevra è stata dedicata ai disegni di Vernet che aveva illustrato e accompagnato con 47 disegni la narrazione de L'Usage du Monde di Bouvier.

(7) F. Laut, N. Bouvier. L'œil qui écrit. Paris, Payot, 2008, pp. 319.

(8) Il materiale fotografico di Bouvier si trova al Musée de l'Elysée di Losanna.

(9) PhilipPe Lejeune, Le Pacte autobiographique, Paris, Éditions du Seuil, 1975, p. 38.
(10) LaUT, $o p$. cit., p. 13. Anche Sartre in un'intervista ebbe ad affermare che la sua biografia di Flaubert, L'Idiot de la famille, era un "roman vrai", cfr. LeJEUNE, op. cit. p. 37

(11) Ph. Lejeune, Je est un autre, Paris, Éditions du Seuil, 1980, p. 70.

(12) Cfr. LauT, op. cit. Ricordiamo che Bouvier nei suoi viaggi usò moltissimo la macchina fotografica, e unisce le foto alle numerose lettere spedite alla famiglia e agli amici per ricostruire la scrittura dei suoi testi. Laut fa rilevare che lo scrittore ginevrino sul suo biglietto da visita aveva fatto imprimere $\mathrm{i}$ termini "Photographe, documentaliste, recherches iconographiques", una sintesi di tutto quello che era stato Bouvier con i suoi testi.

(13) Nicolas Bouvier nacque a Ginevra nel 1929 e qui morì nel febbraio 1998. 
fa da spalla alla prosa e alla poesia del Nostro; diventa quell'occhio del fotografo e dell'iconografo che avrà una parte importante nella produzione letteraria bouvieriana e lo accompagnerà nelle sue numerose ricerche iconografiche, assumendone il ruolo di alter-ego.

Il rapporto tra scrittura e fotografia, l'atto visivo che si materializza nell'immagine fotografica e che in seguito si fa scrittura è per Bouvier, come sottolinea Anne Marie Jaton, ciò che rende «humblement compte du réel», con lo scopo di arrivare alla verità senza intervento soggettivo ${ }^{14}$.

Le tre parti della biografia ${ }^{15}$ con i suoi quattordici capitoli, tutti debitamente intitolati e sottotitolati, scandiscono l'andamento della vita del Nostro dalla nascita all'adolescenza, all'età matura, fino al lento ed inesorabile declino fisico e mettono a nudo molti aspetti significativi e fondamentali della sua esistenza: la madre e la governante molto autoritarie, il padre gentile, colto ma incline alla depressione, l'ambiente della Ginevra intellettuale e accogliente, gli anni della guerra con la chiusura delle frontiere e di una Svizzera costretta al ripiegamento su sé stessa, ai compagni di gioco e di liceo con Thierry Vernet in testa ${ }^{16}$, tutti presenti nell' "esprit" di Bouvier e pronti ad entrare, in modo diverso, nella ricchezza dei suoi scritti ${ }^{17}$. In questi, ultimi è importante sottolinearlo, il viaggio sarà la materia ispiratrice che li permeerà costantemente e che l'autore con un meticoloso lavoro di cesello trasformerà in opere di rara bellezza stilistica e contenutistica. Il viaggio da esperienza unica e individuale si fa altro, lettera e lettura che incontra la collettività dei lettori, suscitando in loro "caleidoscopicamente" una serie di sensazioni, evocatrici di "esperienze" indirette, che si trasmettono senza fine. Lo scrittore-viaggiatore è cosi il tramite e l'interprete di realtà lontane la cui immagine arriva magicamente alla condivisione degli altri ${ }^{18}$.

È proprio la fine del secondo conflitto mondiale che porterà Nicolas e i ragazzi svizzeri verso paesi lontani tramutando quei sogni fatti nella "contemplation silencieuse des atlas, à plat-ventre sur le tapis, entre dix et treize ans" ${ }^{\prime \prime}$, in realtà. Una

(14) Anne-Marie Jaton, Nicolas Bouvier, Lausanne, Le Savoir suisse 2004, p. 66.

(15) La prima parte si intitola Un art de vie, con i sottotitoli: I. Au nom du père (Les ancêtres, Bernard, Auguste); II. Antoinette; III. Les enfants (Le vagabond du Bourneau, Le Haut, Du coté d'Allaman, La rencontre, Le réduit);

IV. Quand partons-nous pour le bonheur? (Un art de vie, Les universités des routes, Été 1948: que de choses vraies, Les affinités électives, Objectif monde, Quand tu aimes, il faut partir (Cendrars). La seconda parte si intitola La moitié du monde coi sottotitoli: V. Etonnants voyageurs, (La Topo, les Tziganes, Fictions?, Le duo, Forni, Swissi! Swissi! La dame de Nick, Fourbus, La fin de l'équipée parfaite); VI. Les Indes galantes, Les miroirs réfléchissent, Mourir de bonheur, Au centre du monde, Tant qu'il y a mouvement, il n'est rien dont désespérer pour demain (Saint-John Perse), Rilke, Agnès, In the occasion of the visit of Mr Nicolas Bouvier, journalist... Milliardaires et musulmans Ashram et absinthe, L'Inde du Sud, Pendant ce temps-là, J'ai tant rêvè de toi, tant marché, parlé, couché avec ton fantôme (Desnos), Amok, Bouvard et Pécuchet, gentlemen adventures, Ils bressolent; VII. La pause ensorcelée: des cris de solitude aux cris de communion. La cent dixième chambre - Le bonheur c'est le vrai talent. Mal au caillou typhon. Rien d'autre que le boulot. Du bagne aux souffles de caresse. La vie militante. Quelque chose de dérangé. Along the gipsy road. Fleuri de fatigue. Destination Japon; Escales/ I VIII. Ma vie pauvre et enchantée; Escales /II IX. Dites-qu'avez-vous vu?; Escales / III $X$. L'exact milieu de ma vie; XI. Le pèlerin vieillit. La terza ed ultima parte si intitola La mélancolie du voyageur, all'interno della quale i capitoli conclusivi portano questi titoli: Un conte ânonné par un âne. Une âme vacante et vagante. S'il est un autre monde.

(16) Thierry Vernet è un amico di Bouvier conosciuto durante gli anni del ginnasio, presso il prestigioso «Collège Calvin» di Ginevra. Entrambi sognano, organizzano e realizzano quel viaggio durato sedici mesi, da giugno 1953 a novembre 1954 , che li portò da Ginevra attraverso la Iugoslavia, la Grecia, la Turchia, l'Iran, il Pakistan, l'Afganistan.

(17) Ricordiamo innanzitutto le tre opere nate dal primo lungo viaggio di Bouvier fatto tra il 1953 e il 1956: L'Usage du Monde, Genève, Droz, 1963. Japon, Lausanne, Rencontre, 1967 (Prix Rambert). Chronique japonaise, Lausanne, L'Âge d'homme, 1975.

(18) Nicolas Bouvier lo fa usando la prima persona, come gli innumerevoli viaggiatori del passato, che descrivono lunghi spostamenti per terra e per mare confondendosi peraltro col protagonista. 
realtà amara dalla quale la gioventù svizzera di quegli anni, per la neutralità del paese, era stata risparmiata, ma che Bouvier scoprirà, quando terminata la guerra, partirà da Ginevra per il lungo, "mitico" viaggio con Thierry Vernet, a bordo della piccola Fiat "Topolino" per arrivare fino a Khyber Pass, in Afghanistan.

Nella Iugoslavia degli anni Cinquanta che soffre ancora della ferite della guerra, Nicolas avrà la prima vera percezione di «ce monde noir et brutal dont ma mère niait l'existence par idealisme» ${ }^{20}$.

Il Nostro, che leggeva già moltissimo per sua propria natura, ci racconta ne $\mathrm{La}$ guerre à buit ans ${ }^{21}$ che il suo «Thesaurus Pauperum» era stato per molti anni non il Petit Larousse dei contadini dell'Auvergne bensì le figurine del cioccolato Nestlé, Peter, Cailler, Kohler, sulle quali, in modo divulgativo, era riportata per soggetti e argomenti la storia mondiale. Il facile accesso ai libri veri e propri ${ }^{22}$, fece di lui una persona coltissima. Così, durante quegli anni difficili di razionamento e di chiusura delle frontiere svizzere, facendo di necessità virtù, il giovane e riflessivo Nicolas si affidò «immobile à grands pas» ${ }^{23}$ a uno spostamento nel tempo prima di poterlo fare nello spazio. Quando venne il momento del viaggio reale, ribalterà, in modo inedito per quegli anni, l'idea del viaggiatore-turista in quella del viaggiatore-apprendista, sprovvisto di tutto e pronto a diventare ricco dell'esperienza e dell'altrui cultura. Come Montaigne, che sottolinea l'importanza del viaggio non come "moda" ma per (...) «rapporter principalement les humeurs de ces nations et leurs façons, et pour frotter et limer notre cervelle contre celle d'autrui $\gg^{24}$ e come Cartesio, che, finiti gli studi, vorrà attingere al «grand livre du monde $»^{25}$, Bouvier trasforma l'avventura del viaggiare in qualcosa che, modificandoci, ci distrugge ed invecchia ma ci arricchisce di altro, di qualcosa che non ha nulla a che vedere con la teoria o la materialità di certe esperienze odeporiche del passato, la cui sintesi è da lui così espressa in una frase di Le Poisson-Scorpion:

On ne voyage pas pour se garnir d'exotisme et d'anecdotes comme un sapin de Noël, mais pour que la route vous plume, vous rince, vous essore, vous rende comme ces serviettes élimées par les lessives qu'on vous rend avec un éclat de savon dans les bordels... Sans ce détachement et cette transparence, comment espérer faire voir ce qu'on a vu? Devenir reflet, écho, courant d'air, invité muet au petit bout de la table avant piper $\operatorname{mot}^{26}$.

I suoi spostamenti cominciano con le brevi escursioni "à vélo" durante il periodo bellico e all'interno della stessa Svizzera seguiti dai viaggi, col ritorno della pace, nella vicina Italia e in Belgio. Allungherà poi il passo andando in Danimarca, Finlandia, Lapponia, Algeria, Spagna e nei Balcani la prima volta nel 195127. Nel 1953, «mollati gli ormeggi» intraprende con l'amico Thierry Vernet quel «grande viaggio» che

(19) N. Bouvier, L'Usage du Monde, op. cit., p. 10.

(20) N. Bouvier, Routes et Déroutes, Entretiens avec Irène Lichtenstein-Fall, op. cit., p. 1259.

(21) Cfr. N. Bouvier, Euvres, op. cit., p. 1241

(22) Ricordiamo che il padre Auguste era stato prima bibliotecario e poi direttore della Biblioteca Pubblica e Universitaria di Ginevra, oggi Biblioteca di Ginevra.

(23) È l'inizio del titolo di un saggio nel quale Bouvier chiarisce l'erroneità di alcuni clichés attribuiti agli Svizzeri e sfata quello della sedentarietà con gli esempi di Divico, Platter, Paracelso. Si tratta di Immobile à grands pas. Éloge de la folie et de quelques Suisses vagabonds, «Cahiers Francophones d'Europe Centre-orientale» 4, Pecs 1994, p. 13.

(24) Montaigne, Essais I, Paris, Folio-Gallimard, chap. 26, p. 226.

(25) Descartes, Discours de la méthode, Paris, Garnier-Flammarion, 2000, p. 39.

(26) N. Bouvier, Le Poisson-Scorpion, Paris, Payot, 1991, p. 46.

(27) Di questi viaggi si hanno testimonianza in alcuni articoli che Bouvier scrisse per la «Tribune de Genève» del 16, 17, 23 e 24 ottobre1948, e per «Le Courrier» dell'11, 18 marzo e del 23 aprile 1959 su richiesta della direzione dei due giornali. 
durerà sedici mesi ${ }^{28}$. L'uno scattando foto e scrivendo articoli, l'altro disegnando e dipingendo: risorse alle quali entrambi dovettero spesso fare ricorso per procacciarsi quel minimo indispensabile, necessario alla sopravvivenza e al proseguimento del viaggio.

In quegli anni, pur densi di tensioni politiche internazionali, la guerra fredda tra URSS e Stati Uniti prima e la crisi di Cuba poi, nulla avrebbe potuto far loro sospettare che il nuovo millennio ci avrebbe costretti tutti ad un viaggio molto meno rassicurante.

I tristi avvenimenti dell'11 settembre hanno fatto crollare oltre che le "Torri", anche quel piacevole stato d'animo, intriso di sogni e curiosità, che spingeva tanti giovani nell'età delle illusioni ad andare fuori dai confini di casa propria per completare una formazione che solo il viaggio poteva dare ed hanno fatto conoscere in modo violento luoghi, uomini, donne, vecchi e bambini di una vasta, affascinante regione, che se pur lontana e da pochi esplorat $\mathrm{a}^{29}$, è diventata sempre più nota, più vicina e allo stesso tempo sempre più temuta perché teatro di violenze inaudite.

Il materiale manoscritto che François Laut ha selezionato con estrema cura durante i suoi diciotto mesi di ricerca presso gli Archivi della Biblioteca di Ginevra, gli ha permesso di restituire quegli elementi indiscutibilmente importanti per il profilo fisico, psicologico e letterario di Nicolas Bouvier che i lettori de L'œil qui écrit possono apprezzare molto facilmente. Senza stravolgere l'evidenza interna delle informazioni manoscritte, Laut in questa biografia non perde di vista la realtà, non cade nel romanzesco ma restituisce un ritratto autentico dello scrittore ginevrino come egli stesso ha affermato nella prefazione ${ }^{30}$. Nella presentazione di Bouvier, Laut segue l'esempio dell'Idiot de la famille di Sartre ${ }^{31}$ e va anche oltre la storia dei genitori, risalendo addirittura ai trisavoli e bisavoli del Nostro; ne traccia così un profilo importante che calzerà con tutte le sue specificità soprattutto con quella di "grand conteur" sul quale prevarrà lo scrittore. Un episodio che il Nostro ci racconta ne La Guerre à buit ans ci aiuta a capire il suo dramma di fanciullo sottoposto alla rigida educazione calvinista della madre da una parte e a quello della governante prussiana Bertha dall'altra. E dunque una sera, durante uno dei suoi soggiorni estivi ad Allaman presso la residenza dei nonni materni, Nicolas deciso a «échapper à la tirannie de la governante prussienne qui régnait sans partage sur la maison et nous terrorisait $\aleph^{32}$, e desideroso di poter rimanere più a lungo tra gli adulti e conoscere «quantité de choses que j'ignorais» ${ }^{33}$, decise di rispondere alla domanda di uno degli ospiti su ciò che succedeva in Cina alla fine del XIII secolo. La risposta esatta del piccolo Bouvier lasciò gli astanti stupefatti ed egli si guadagnò il permesso di rimanere tra loro.

E la prima rivincita del futuro scrittore sulla "sadica" governante Bertha che «purgeait sans pitié au clystère de savon noir le chétif que j'étais» ${ }^{34}$. L'episodio e il travaglio che precede questa presa di parola, ci riporta alla mente la «plaie profonde», «toujours cachée» evocata da Sartre quando allude alla balbuzie di Flaubert; un

(28) Thierry parte nel giugno1953 e precede Nicolas in Iugoslavia. Quest'ultimo terminati gli esami all'università lo raggiungerà a Belgrado alla fine di luglio. Il loro viaggio durerà sedici mesi dopodiché i due si separeranno. Thierry si farà raggiungere dalla fidanzata e si sposeranno a Ceylon, Nicolas si dirigerà verso il Giappone dove soggiornerà fino $\mathrm{al}$ 1956.

(29) Ricordiamo a questo proposito che anche la grande viaggiatrice svizzera Ella Maillart ci ha lasciato numerose opere basate sui resoconti dei suoi viaggi cui unisce anche documenti fotografici. Della
Maillart citiamo Des monts célestes aux sables rouges, Paris, Petite Bibliothèque Payot, 1934, in cui riferisce di un'impresa in oriente quasi impossibile.

(30) LAUT, op. cit. p. 13. «Intus et in cute» pour reprendre l'exergue des Confessions de Jean-Jacques Rousseau.

(31) Jean-Paul Sartre, L'Idiot de la famille, Paris, Gallimard, 1971.

(32) N. BOuvIER, La guerre à buit ans in Euvres, op. cit., p. 1240.

(33) Ibidem.

(34) Ibidem, p. 1242. 
difetto nascosto, represso che diventerà il volano di quella monumentale produzione letteraria che fa dell'«idiot de la famille» il creatore del romanzo moderno. Bouvier quella sera afferma il proprio "io" di fronte alla società raffinata e colta dell'entourage familiare, riprendendo una volta per tutte quel controllo della sua personalità soffocata per anni da una delle «figures les plus détestées de mon enfance, le gardechiourme prussien Bertha qui avait fait de moi - j'étais le plus jeune - avec la lâche complicité des adultes son bouc émissaire et souffre-douleur favori» ${ }^{35}$.

Dalla spensierata partenza («...comme les vrais voyageurs de Charles Baudelaire, ils partaient pour partir, "coeurs légers, semblables au ballons" $»^{36}$ ), alla fine «de l'équipée parfaite, à deux» ${ }^{37}:$ dal rapporto quasi sempre sereno dei due amici, alle piccole divergenze dovute alla naturale differenza dei caratter ${ }^{38}$, dagli amori di Nicolas, ai numerosi amici incontrati sul loro cammino ${ }^{39}$, questa biografia non trascura nessun dettaglio sul Nostro. Lo spostamento nello spazio sulla terra ferma a bordo della piccola Fiat "Topolino" "lascerà il posto, nella parte centrale della biografia, i capitoli VII e VIIII, all'elemento "liquido" che pur non interrompendo il movimento fisico del viaggiatore, lo descrivono da un altro punto di vista: il mare.

Il soggiorno in Giappone, il primo di una serie di viaggi in questo paese, obbligherà il Nostro, ora che è rimasto solo, e a più forte ragione ${ }^{41}$, a sopperire ai bisogni materiali. Riprenderà cosi l'abitudine di scattare fotografie per venderle, affiancandola alla redazione di articoli che lo rafforzeranno in quella antica idea divenuta poi ossessione, la pubblicazione di un libro:

Ce voyage ce cadeau du monde va me devorer me tuer, m'étouffer comme une lierre - il faut le comprendre - il en a droit, je suis endetté jusq'au trognon, je dois au monde une énorme explosion, un coup de cymbale magistral. Et je me mets au travail, avec quelle peine. ${ }^{42}$

Tornerà di nuovo nel paese del Sol Levante nel 1964 con la moglie Éliane che ha sposato nel 1958 e col primo figlio Thomas ${ }^{43}$ e nel 1970 per l'esposizione universale di Osaka ${ }^{44}$.

Questi viaggi successivi sottolineano un cambiamento di prospettiva: al singolo compagno subentrano la moglie e i figli, alla spensieratezza degli anni giovanili in compagnia di Thierry una progressiva maturazione e riflessione che inevitabilmente portano a modificare l'osservazione delle cose e degli esseri visti precedentemen$\mathrm{te}^{45}$. Malinconia e pessimismo cominciano a farsi largo nella fase di mezzo della vita dell'autore ginevrino aprendo una nuova dimensione al suo rapporto con la scrittura.

(35) Ibidem, p. 1244.

(36) LAUT, op. cit., p. 76.

(37) Ibidem, p. 94

(38) Scopriamo un Thierry Vernet più indeciso sull'itinerario del viaggio, soprattutto quando le difficoltà lo mettono a dura prova, e un Nicolas molto determinato nel voler continuare.

(39) LAUT, op. cit., pp. 86-90.

(40) È il terzo protagonista de L'Usage du Monde; regalo paterno per aiutare Nicolas a raggiungere l'università dopo un incidente occorsogli durante il servizio militare. Quest'automobile aggiunge altra singolarità a questo mitico viaggio, e diventa l'anima e il respiro dello spostamento nello spazio, mezzo di trasporto prezioso e come tale bisognoso di attenzione e manutenzione.

(41) Thierry si separa da Nicolas a Khyber Pass.
La fidanzata Flo lo raggiungerà e si sposeranno a Ceylon.

(42) Si legge nel manoscritto Cahier 4 noir (Genève, 1958-1960) f. 1 riguardante il Plan, textes et notes di preparazione all'Usage du Monde. Un progetto che abita il Nostro sin dall'inizio del lungo viaggio intrapreso nel luglio 1953.

(43) Il secondo figlio Manuel nascerà durante questo soggiorno a Tokyo il 12 dicembre 1964.

(44) Anche questa volta sarà accompagnato dalla moglie Éliane.

(45) In effetti il Nostro aveva effettuato nel periodo bellico qualche "randonnées à vélo" all'interno della Svizzera; finita la guerra verrà in Italia, a Firenze; poi sarà la volta del Belgio e di molti altri paesi europei: vedi supra. 
È la dimensione poetica ${ }^{46}$ quella che avrebbe voluto praticare se la vita non gli avesse imposto le sue regole pratiche, quella che si libererà in lui sempre più forte dopo le crisi depressive. Ma vi è anche un Bouvier musicista, che rinuncia alla carriera di pianista, un amatore di ritmi che impregneranno non solo i suoi viaggi ma anche gli scritti; dai ritmi tzigani dell'Usage du Monde, al Quatuor di Debussy di Le PoissonScorpion, ascoltato fino all'ossessione come a volersi esorcizzare dall'incubo in cui era caduto a Ceylon, alle note della musica giapponese e ai ritmi orientali. Letteratura, musica ma anche arte popolare ripresa per celebrare il settecentesimo anniversario della Confederazione nell'Art populaire: Giacometti, Amiel, Rougemont, Roud, Frisch, Walzer, in cui è illustrata l'ars moriendi, mentre la sua arte di vivere sarà espressa in Routes et déroutes ${ }^{47}$.

Nicolas Bouvier è anche l'esportatore nel mondo dell'immagine dello svizzero errante ${ }^{48}$. Immagine sulla quale ritornerà nel suo ultimo lavoro L'Échappée belle. Éloge de quelques pérégrins ${ }^{49}$, che sarà celebrata anche se un po' in ritardo. E un omaggio agli svizzeri nomadi e a qualche altro viaggiatore stupefacente come Gaulis, Pestelli e Godel, ci dà anche qualche chiave della sua scrittura, pagando il debito a tutti quelli che lo hanno preceduto. Gli svizzeri appaiono come dei grandi viaggiatori, come il popolo più nomade d'Europa che batte addirittura gli irlandesi; nomadismo che non è nato ieri ma risale a più di duemila anni fa, al 58 a.C. Dall'avventura di Divico alla sconfitta di Bitracte, dal Drang nach Süden alla battaglia di Marignan, la Svizzera nomade è passata in rassegna fino ai "claustrofobici" Platter e Paracelso, diventati vagabondi, senza escludere Rousseau e le sue promenades.

Questo elogio della Svizzera romanda in movimento sembra quasi essere un tentativo da parte di Bouvier di voler cancellare lo stereotipo del paese stabile, sedentario, razionale, industrioso, interamente dedito al risparmio, al settore terziario e al fucile militare. Una rassegna di duemila anni di storia svizzera dove sono convocati tutti gli Elveti: esiliati, volontari, celebri ed anonimi, aristocratici e mercenari per arrivare a Blaise Cendrars e spiegare che oltre al mercenariato può esserci anche tanto desiderio di conoscenza. Gli esempi che Bouvier cita ci riportano proprio a lui grande viaggiatore ma anche al sedentario, per necessità, che nella dialettica del «s'attacher et s'arracher» ha basato tutta la sua scrittura e che lo ha accompagnato fino agli ultimi giorni. Per Bouvier senza il viaggio non sarebbe nata la sua scrittura e senza la scrittura il viaggio non si sarebbe in alcun modo materializzato agli occhi dei lettori.

Oggi egli riposa nel piccolo cimitero di Vandœuvres a due passi dal "vieux toit", la casa abitata prima coi genitori e poi per tutta la vita con la moglie e i figli. Casa dalla quale si allontanava, «s'arrachait» per i suoi numerosi ed interessanti viaggi e nella quale tornava a «s'attacher $\aleph^{50}$ per poter trasformare quel tesoro visivo delle esperienze dei viaggi nell'oro della sua splendida e levigatissima parola.

FRANCESCA MORALE

(46) Sono gli scrittori Svizzeri Chessex e Chappaz con l'amico Thierry Vernet che applaudono alla sua vena poetica.

(47) Si tratta di un'intervista biografica e tematica realizzata dalla giornalista Irène Lichtenstein-Fall.

(48) Quest'immagine sarà celebrata in ritardo; rimandiamo al ben noto saggio del Nostro in cui è sfatato il cliché della sedentarietà elvetica: Immobile à grands pas. Éloge de la folie et de quelques Suisses vagabonds, «Cahiers francophones d'Europe Centre-Orientale» 4, 1994, pp. 7-12.

(49) N. Bouvier, L'échappée belle. Éloge de quelques pérégrins, Genève, Éditions Métropolis, 1996.

(50) I termini "s'arracher" e "s'attacher" sono stati ripresi da una definizione che Bouvier dà della vita nomade in Routes et Déroutes, Entretiens avec Irène Lichtenstein-Fall, in Euvres op. cit., p. 1290. 